for only 20-30 sec. with thallium, owing to the high volatility of its fluorides. In no instance has admixture with up to 5 per cent of other compounds been found to interfere with the effects of lead and bismuth. No permanent modiflcation of the surface of the platinum can be postulated, since no anomalous behaviour is observed when pure cryolite is fused in a platinum crucible from which a non-wetting melt has been cleaniy detached.

Although the precise limits have not been determined, it is known that as little as 1 part of lead or bismuth in 5,000 can cause this nonwetting effect with cryolite. The limits are higher for potassium
wern cryolite ( $\mathrm{K}_{3} \mathrm{AlF}_{\mathrm{s}}$ ), being approximately 1 part in 20 for lead and 1 in 400 for bismuth.

Synthetic cryolite, prepared from hydrofluoric acid and tri-sodium aluminate solutions in lead vessels, is non-wetting; but, when made in rubber or platinum ware, the synthetic material cannot be disOther

Other melts, such as sodium sulphate, sodium fluoride, sodium tungstate and potassium sulphate, are affected like cryolite, while sodium chloride, and metaphosphate are not. Lead fluoride itself until nearly twice its weight of cryolite has been added.

Although no explanation of these phenomena is proffered at the present juncture, it is felt that the unusual nature of the observations makes it desirable to place them on record.

T. R. SCOTT.

Division of Industrial Chemistry,

Council for Scientific and Industrial Research, Melbourne.
Sept. 25 .

\section{Approximate Expression for Loss of Heat from Exposed Surfaces}

THE standard expression for the loss of heat from exposed surfaces by radiation and convection is:

$$
H=1 \cdot 36 E \times 10^{-12}\left(T_{1}^{4}-T_{2}^{4}\right)+5 \cdot 0 \times 10^{-5}\left(T_{1}-T_{2}\right)^{5 / 4},
$$

where $\boldsymbol{H}$ is loss in cal. $/ \mathrm{cm}^{2} / \mathrm{sec}$. : $\boldsymbol{E}$ is emissivity $(E=1.0$ for black body); and $T_{1}, T_{2}$ are absolute temperatures of surface and surroundings.

This expression is clumsy to solve and is usually employed in the form of diagrams or tables. It can be closely approximated for practical purposes up to $350-400^{\circ} \mathrm{C}$. above room temperature for any emissivity by an expression :

$$
\boldsymbol{H}=\boldsymbol{A \theta}+\boldsymbol{B} \theta^{2} \text {, }
$$

where $\theta$ is excess of body temperature above surroundings.

Values for $A$ and $B$ have been found for various assumed values of $E$ using $\theta=100$ and $\theta=300^{\circ} \mathrm{C}$, that is, $T_{1}=385$ and 585 , and $T_{\mathrm{s}}=285$.

$$
\begin{aligned}
& E=1.00 \\
& \begin{array}{l}
0.75 \\
0.50
\end{array} \\
& \begin{array}{l}
0 \cdot 50 \\
0 \cdot 25
\end{array} \\
& \begin{aligned}
A= & 1.96 \\
& 1.80 \\
& 1.64 \\
& 1.48
\end{aligned} \\
& B=1.71 \times 10^{-6} \\
& 1 \cdot 34 \\
& \begin{array}{l}
0.98 \\
0.62
\end{array} \\
& \begin{array}{l}
0 \cdot 62 \\
0 \cdot 25
\end{array}
\end{aligned}
$$

$1.96 \times 10^{-4}$
1.80
1.64
1.48
1.33

It so happens that these

$$
B=10^{-4}(1 \cdot 33+0 \cdot 63 E) \theta+10^{-6}(0 \cdot 25+1 \cdot 46 E) \theta^{2}
$$

is a close approximation over the prescribed range.

The use of this expression enables problems of various types to be solved as a simple quadratic equation. For example, the calculation of the heat loss through a body exposed to a known temperature on one side and cooled by natural convection and radiation on the other comes into this category, and it is also simple to find the temperature reached leads to estimates of the temperatures in these cases which are more than sufficiently accurate for practical purposes.

Chance Brothers, Itd.,

Glass Works,

$$
\text { Dec. } 11 \text {. }
$$

\section{'Industrial' Separation of Isotopes}

IT has been shown ${ }^{2}$, and confirmed by other workers ${ }^{2}$, that carbon tetrachloride evaporated in the vicinity of a charged Van de Graaff electrostatic generator almost doubled the voltage of the spherical electrode. The phenomenon was attributed to the difference of mobility of the molecules of carbon tetrachloride and that of the average air molecule, and the electro-affinity of the former.

The effect is most striking on sharp edges and points on the electrodes. The corona effect appearing on them is instantly suppressed in the presence of carbon tetrachloride vapour.

When the surrounding gas mixture is ionized, there is a double When the surrounding gas mixture is ionized, there is a double
flow of ions to and from the charged electrode. The mobility of ions is inversely proportional to the square root of their molecular weights. Thus the mobility of carbon tetrachloride ions is about one third of that of other lons present in air. A layer of increased concentration of that of other lons present in air. A layer of increased concentration
of the heavier ions is thus formed in contact with the electrode. The of the heavier ions is thus formed in contact with the electrode. The concentration is sufficiently high to change the behaviour

The increased tension of the generator is due to the sudden lowered rate of loss of charges.

It is thought that the Van de Graaff or Pauthenier high-tension generators might be used effectively to separate gas mixtures into their constituents in accordance with their molecular weights. The large surface of the electrodes in question and the considerable voltage
available suggest the possibility of separation on an 'industrial' scale.
Certain modifications of the electrodes when used for the purpose of separation should increase the amounts of material that could be dealt with as well as the separation factor.

The method is not limited to gases only.

H.M.A./S.E.E.,
Fairlie, Ayrshire.

M. FELDENKRAIS.

I Dec. 17.

1 Joliot, F., Feldenkrais, M., and Lazard, A., C.R. Acad. Sci., Paris, 202,291 (1936)

"Stranathan, J. D., "The Particles of Modern Physics".

Feldenkrais, M., thèse de doctorat (Paris, Nov. 1945).

\section{A New Anti-Vibration Support}

IN his investigation of the optical constants of liquid metals, Aster ${ }^{1}$ placed marble slabs on sand to obtain a shock-proof arrangement. This proved impracticable in my work. The laboratory is situated on the second floor of a large five-storey building. The vibrations of the latter trolley-buses passing at no great distance, and the runing deving of an electric motor in the laboratory made it ill these factors on a mercury surface, and, at the same time, be rigid enough to carry a small furnace.

The application of newspaper paddings, sawdust, cycle- and cartyres, large 'india-rubbers', books, heavy stone-slabs, sand and cork, separately or combined, was fruitless.

Finally, the cycle-tyre was cut into sections about one and a half inches long. The straight edges were gripped, turned inside out an pinned together. They provided spring elements of great elasticity. Square cork slabs (6-in. edges) were then piled on top of each other until a height of about two inches was reached. On these there were placed eight of the above rubber springs, which formed approximately a circle. Another two-inch layer of cork was laid on the springs and a retort-stand placed on the former. Two other similar supports were erected. Three eyes, screwed into three lengths of curtain-wire (18 in.), were flxed to a wooden board ( 12 in. $\times 15$ in.), and this arrangement was then suspended from the retort-stands. A heavy stone-slab was carefully lowered on to the board and the basin flled with mercury was put on the slab.

wibrations to the mercury via the floor proved to be futlle.

An effort was made to eliminate the rubber springs, but it was soon found that they formed a crucial part of the supports. It is felt that the many degrees of freedom-the thickness of the cork, the length of the (expanding) curtain-wires, the weight of the stone-slabenable the arrangement to meet a large variety of conditions. In this, as in its economic aspect, the simple set-up is superior to various factory-made products.

South-West Essex Technical College,

ROBERT WEIL.

$$
\text { Forest Road, Lon }
$$

${ }^{1}$ Aster, Phys. Rev., 20, 349 (1922).

\section{Red Dwarf Stars}

THE characteristics of a red dwarf star are: low luminosity (say, not more than one tenth that of the sun), small mass (say, not mor than three quarters that of the sun) and high density (perhaps 30-100 times the density of the sun).

That stars of small mass should be of low luminosity is not surprising, but that the density should be as high as it is has always been somewhat of a puzzle. The purpose of the present note is to offer what appears to be an explanation of the high density.

The energy radiated by the sun and by other stars of the main sequence is believed to be due chiefly to the conversion of hydrogen into helium, the reaction taking place in the central core, which is assumed to reach a sufficient temperature to make this reaction possible. Now when a cloud of scattered material first contracts to form a star or planet, the hydrogen which it contains tends to collect in the
outer layers rather than in the core, and, in the case of the planets, this distribution of material is permanent.

In the case of stars belonging to the main sequence, it seems to be necessary to assume that the general conditions in the interior of the star are such that convection currents occur which are of sufficient importance to convey hydrogen in adequate amounts from the outer layers to the central core, although this does not imply that the convection currents are of sufficient magnitude to be the dominating factor in determining the distribution of temperature.

It would be unreasonable to assume that all stars the mass of which is appreciably less than that of the sun are deflcient in hydrogen and what suggests itself is that the red dwarfs probably contain and what suggests itself is that the red dwarfs probably contain a normal proportion of hydrogen, but that convection currents in these stars have never been of suffeient magnitude to convey the hydrogen in adequate amounts from the outer layers to the central core. Presum ably chere is a critical value of the mass which determines the actual course of stellar evolution. When the mass of the star is less than this critical value (say three quarters the mass of the sun), the hydrogen
which the star contains never reaches the core in sufficient quantity which the star contains never reaches the core in sufficient quantity to initiate and sustain the process of transforming hydrogen into
helium, and dynamical equilibrium is secured only by contraction involving comparatively high densities.

On this theory the red dwarfs are not stars of great age which have consumed their substance in riotous living; they are simply feeble things which have never been able to make use of the supplies of fuel which they actually possess.

Cherbury, Booterstown, K. E. EDGEWORTH. 